\title{
Characterization of agarose microgels for nutraceuticals encapsulation
}

\author{
A. Ellis and J. C. Jacquier *
}

School of Agriculture, Food Science and Veterinary Medicine, University College Dublin, Belfield, Dublin 4, Ireland

corresponding author: jean.jacquier@ucd.ie

Agarose based microgels were investigated as potential encapsulation delivery systems for a wide range of lipophilic nutraceuticals in food. The main objective was to compare the rheology of angular and spherical microgels of varying sizes. Results show that the flow of microgel suspensions was strongly dependant on both shear rate and microgel volume fraction, and was reminiscent of concentrated dispersed systems with a sharp rise in viscosity near the maximum packing fraction.

\section{Materials and Methods}

Angular microgel suspensions were prepared by shearing 5\% bulk agarose gels (Gelagar CT 1.0 from B\&V, Italy) using a Silverson L4RT rotor/stator mixer (Silverson Machines Ltd, UK) equipped with a fine emulsion screen at $8000 \mathrm{rpm}$ for up to $16 \mathrm{~min}$. Spherical beads were prepared by a water in oil emulsion technique in the same size range as angular particles. Particle size analysis for both types of microgel suspension was carried out using the Malvern Mastersizer S (Malvern Instruments Ltd, UK) with 300RF range lens and Small Volume Dispersion Unit. Steady Stress Sweep tests were carried out using a SR-2000 (Rheometric Scientific) with Couette geometry. Particle phase volume $(\phi)$ of all samples was established using the blue dextran method (Lopez et al., 2004).

\section{Results and Discussion}

Figure 1 shows the size distribution of the microgels. Despite the fact that the size distributions seem narrower for the large gel particulates than for the small ones on this figure, smaller particles were found to have a smaller polydispersity index than the large ones, defined as the ratio of the volume average $\mathrm{D}[4,3]$ to the surface average diameter $\mathrm{D}[3,2]$ (Table 1). This could be explained by a substantial amount of relatively small particles $(<100 \mu \mathrm{m})$ for both large gels which, although represents a tiny proportion of the volume $\%$, impacts negatively on the monodispersity.

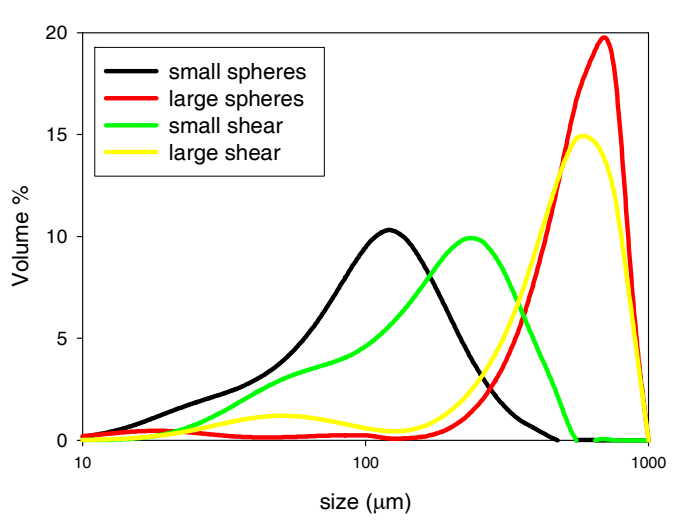

Figure 1. Particle Size Distribution of Microgels

\begin{tabular}{|c|c|c|c|c|}
\cline { 2 - 5 } \multicolumn{1}{c|}{} & $\begin{array}{c}\mathrm{D}[3,2] \\
(\mathrm{mm})\end{array}$ & Polydispersity & $\Phi_{\mathrm{m}}(\%)$ & {$[\eta]$} \\
\hline $\begin{array}{c}\text { Small } \\
\text { Spheres }\end{array}$ & 71 & 1.64 & 66 & 3.0 \\
\hline $\begin{array}{c}\text { Large } \\
\text { Spheres }\end{array}$ & 246 & 2.25 & 37 & 2.4 \\
\hline $\begin{array}{c}\text { Small } \\
\text { Sheared }\end{array}$ & 113 & 1.67 & 46 & 4.1 \\
\hline $\begin{array}{c}\text { Large } \\
\text { Sheared }\end{array}$ & 229 & 2.11 & 30 & 3.2 \\
\hline
\end{tabular}

Table 1: Characteristics of the microgels

The flow behaviour of the microgel suspensions under constant shear stress was studied in order to evaluate the dependency of viscosity on particle phase volume. The flow curve shown on Figure 2 in the case of small spherical beads was typical of the ones found for the 
other microgel suspensions. At low $\phi$, all microgel suspensions are essentially Newtonian, however as phase volume increases towards maximum packing fraction $\phi_{\mathrm{m}}$, a more complex flow behaviour is observed. After an apparent yield stress and strong shear-thinning behaviour, these concentrated dispersions show high shear plateau with slight shear thickening behaviour for the most concentrated ones. As shear stress increases further, wall slip occurs, as revealed by a sudden drop in viscosity. The relationship between the particle phase volume $\phi$ and viscosity (taken as high shear plateau viscosity) can be expressed using the Kreiger-Dougherty (1959) equation, $\eta=\eta_{\mathrm{f}}\left(1-\phi / \phi_{\mathrm{m}}\right)^{-[\eta] \phi \mathrm{m}}$, where $\eta_{\mathrm{f}}$ is the suspending fluid viscosity, $\phi$ is the volume fraction of the solid particles, $\phi_{\mathrm{m}}$ the maximum packing fraction and $[\eta]$ the intrinsic viscosity. The derived values of $[\eta]$ and $\phi_{\mathrm{m}}$ for all microgels are grouped in Table 1 and Figure 3 shows both the experimental points and the KriegerDougherty theoretical treatment for each microgel. It is obvious from these results that both size and shape influence the flow behaviour of the particulate dispersions. The maximum packing fraction $\phi_{\mathrm{m}}$ is negatively influenced by the isotropy of the microgels, as both sheared and therefore angular gels show lower $\phi_{\mathrm{m}}$ than the spherical ones. On the other hand, the intrinsic viscosity [ $\eta]$, which is intimately related to specific particle resistance to flow and largely depends on particle shape, is positively correlated to the isotropy of the microgels, with larger values found for sheared than for spherical beads.

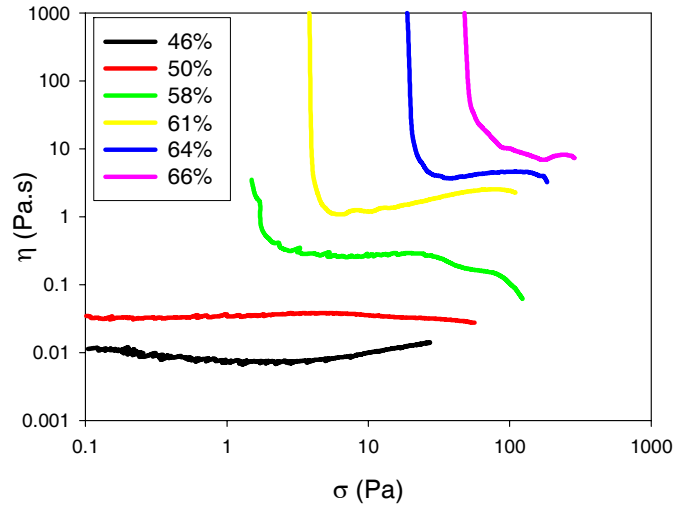

Figure 2. Steady Shear Stress for small bead microgel suspensions

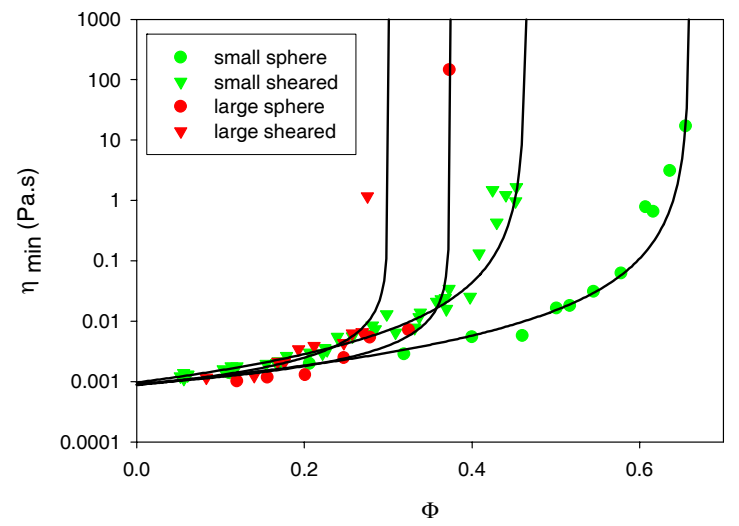

Figure 3. Relationship between phase volume and viscosity

The influence of size on the Krieger parameters is more complex, as either due to higher deformability of the larger gels or higher polydispersity, both responsible for a substantial decrease in $\phi_{\mathrm{m}}$. Although the influence of size on [ $\left.\eta\right]$ is difficult to interpret, the impact on $\phi_{\mathrm{m}}$ is dramatic, as can be seen in figure 3 . In the case of spherical beads at $40 \%$ volume fraction, while the small beads will impart only a small increase in viscosity to water, the large beads dispersion does not flow and is solid-like.

\section{Acknowledgements}

This research has been part-funded by grant aid under the Food Institutional Research Measure, which is administered by the Department of Agriculture and Food, Ireland.

\section{References}

Adams S., Frith W.J., and Stokes J.R. (2004) J.Rheol 48(6), 1195-1213.

Kreiger I.M. and Dougherty T.J. (1959), Trans. Soc. Rheol., 3, 137-152.

Lopez E.C., Rolee A., and Le Meste M., (2004), Starch., (56), 576-581. 